The first 1000 days of life are a period of unique sensitivity and plasticity during which critical cognitive abilities are formed. Routine developmental screening tools aim to identify infants who would benefit from early intervention. While these tools have been validated for detecting children with more severe neurodevelopmental disorders, their ability to identify the larger proportion with below average cognitive function has not been sufficiently explored. The aim of this study was to examine the validity of the Ages and Stages Questionnaire (ASQ), for identifying children with later below average cognitive function.

The study population $(n=8260)$ is formed from two national cohort studies, the Growing Up in Ireland (GUI) Infant cohort $(n=7,444)$ and the Cork BASELINE cohort $(n=816)$. The ASQ was completed at 8 months and 24-27 months respectively. Cognitive assessments were performed at age 5. Those scoring $<1$ standard deviation (SD) below the mean were categorised as below average cognitive function. Applying the currently used onward referral criterion (one fail in any domain) the sensitivity, specificity, positive and negative predictive values of the 8- and 24-27- month ASQ for detecting children with later below average cognitive function were calculated.

In the GUI cohort $\mathrm{n}=905$ participants $(12.5 \%)$ had scores $<1 S D$ below the mean on the Picture Similarities Scale. In the BASELINE cohort $n=101$ participants $(13.4 \%)$ had an IQ $<1 S D$ below the cohort mean. Applying the currently used onward referral criterion (failing in any one domain in the ASQ), the sensitivity of the 8-month ASQ for detecting children scoring $<1 \mathrm{SD}$ below the mean on the Picture Similarities Scale at age 5 was $16.4 \%$ (95\% CI 14.0-19.0). The specificity was $92.0 \%$ (95\% CI 91.3-92.6), with a positive predictive value (PPV) of $22.6 \%$ (95\% CI 19.5-26.0) and a negative predictive value (NPV) of $88.5 \%$ (95\% CI 87.7\%-89.2\%).

In the BASELINE cohort $\mathrm{n}=468$ participants completed the 24-month ASQ and $n=316$ the 27-month ASQ. Applying the same onward referral criterion to the 24- and 27- month ASQ combined, the sensitivity for detecting those with an IQ $<1$ DD below the cohort mean was 20.8\% (95\% CI 13.6-30.2) and the specificity was $91.1 \%$ (95\% CI 88.6-93.2).

The ASQ has a low sensitivity for identifying children with below average cognitive function at age 5. The findings of this study suggest that if we are to intervene early in the developmental trajectory for children with below average cognitive function alternative methods of identifying high risk infants are needed.

\section{EMOTIONAL BEHAVIOURAL DEVELOPMENT IN CHILDREN WITH BELOW AVERAGE COGNITIVE FUNCTION}

Andrea Bowe*, Deirdre Murray, Anthony Staines. INFANT Research Centre, Cork University Hospital, Ireland

\subsection{6/archdischild-2021-europaediatrics.474}

Children with below average cognitive function represent a substantial yet under-researched population for whom academic and social demands, which increase in complexity year by year, pose significant challenge. Effects on emotionalbehavioural development (EBD) are not well understood. The aim of this study was to compare trajectories of EBD for children with and without below average cognitive function.
The underlying hypothesis was that trajectories of EBD would differ between groups, with divergence occurring as children are subject to increasingly complex cognitive demands.

Participants consist of 7,000 children and caregivers who completed the Growing Up in Ireland survey at age 3,5 and 9 years. Cognitive function was measured at age 3 using the Picture Similarities Scale. A t-score 1-2 standard deviations below the mean was categorised as below average cognitive function $(n=767)$, and scores above this categorised as average cognitive function $(n=6418)$. EBD was measured using the Strengths and Difficulties Questionnaire (SDQ) at age 3,5 and 9. Repeated measures ANOVA was used to examine the difference between the overall group means across the repeated measures of SDQ, the SDQ change over time, and the interaction between cognitive group and change over time. Further analysis of trajectories was performed using latent growth curve analysis.

Compared to those with average cognitive function, a significantly higher proportion of children with below average function were male $\left(61.8 \%\right.$ v $38.2 \%, \chi^{2}(1, \mathrm{~N}=7134)=$ $42.07 \mathrm{p}<0.001)$, born to a single parent family $(22.4 \% \mathrm{v}$ $\left.14.7 \%, \chi^{2}(1, \mathrm{~N}=7134)=23.15 \mathrm{p}<0.001\right)$, and had a parent who smoked $\left(35.6 \%\right.$ v $25.8 \%, \chi^{2}(1, \mathrm{~N}=7134)=33.23$ $\mathrm{p}<0.001)$. Children with below average cognitive function had significantly higher mean total SDQ scores at all ages. Repeated measures ANOVA demonstrated a significant groupby-time interaction effect $(\mathrm{F}(2,7182)=4.649, \mathrm{p}=0.010)$. The mean difference (MD) in SDQ between cognitive groups increased over time (MD Age 3:0.87, 95\% confidence interval (CI) 0.53-1.21, Age $9 \mathrm{MD}: 1.49,95 \%$ CI 1.08-1.91). For those with average or above cognitive function the overall SDQ decreased between age 3-9 (MD: -0.49 95\% CI -0.65-0.33). This decrease was not seen for those with below average function, who had a non-statistically significant increase in SDQ between age 3-9.

Children with below average cognitive function experience higher and worsening mean SDQ scores throughout childhood. A scalable method of early identification of children at risk for below average cognitive function should be a research priority for public health, enabling early intervention for cognitive and adaptive outcomes.

\section{ASSISTED REPRODUCTIVE TECHNOLOGY TECHNIQUES AND RISK FOR NEURODEVELOPMENTAL DISORDERS}

Lucija Bajkovec*, Ines Begović, Ana-Marija Đaković, Milan Milošević, Urelija Rodin, Aida Mujkić. Institute of Emergency Medicine of Medjimurje County

10.1136/archdischild-2021-europaediatrics.475

Assisted reproductive technology (ART) techniques are becoming more common nowadays, due to the growing incidence of infertility. Such pregnancies are more often multiple, which carries the risk of premature birth and lower birth weight and, accordingly, a higher risk of occurrence of neurodevelopmental disorders. Aside from the before mentioned, other known risk factors for neurodevelopmental disorders include chorioamnionitis, gestational diabetes, and respiratory distress. The aim of the study was to compare the prevalence of risk factors for neurodevelopmental disorders between two groups of children born preterm, those conceived through ART and those conceived naturally. 
This was a retrospective study based on the data of births collected by Croatian Institute of Public Health for the year 2018. The study included a total of 2233 children born before week 37 of gestational age, 181 of which were conceived through ART. The prevalence of pathological conditions during pregnancy, delivery, and at birth, known as neurodevelopmental risk factors, was compared between children conceived through ART and those conceived naturally. The Mann-Whitney $U$ test was used to perform data analysis.

Pathological conditions in pregnancy were recorded in $82.3 \%$ of children conceived by ART, compared to $58.4 \%$ of children conceived naturally $(\mathrm{p}<0.001)$. The most common disorders during pregnancy are twin pregnancy $(2.8 \%)$, chorioamnionitis $(2.8 \%)$, and retarded fetal growth $(2.8 \%)$. On the other hand, in the group of naturally conceived neonates, the most common diagnoses were retarded fetal growth $(3.5 \%)$ and gestational diabetes (3.0\%).

It was observed that the share of children with pathological conditions was significantly higher in the group of those conceived by ART (92.2\%) compared to the second group $(84.1 \%)$ of children $(\mathrm{p}=0.003)$. The diagnoses that predominated among the ART conceived after delivery were neonatal respiratory arrest (24.9\%) and low birth weight (14.9\%). Additionally, the mean birth weight was significantly lower in the group of children conceived by ART (2170.77 grams), in comparison to another group (2307.68 grams, $\mathrm{p}=0.015)$. Nevertheless, groups of neonates did not differ significantly in postnatal mortality $(\mathrm{p}=1.00)$.

Newborns conceived by ART are more likely to suffer from conditions that represent risk for factors for neurodevelopmental disorders. Neonatal respiratory arrest especially represents the risk for the neurodevelopmental disorders. The results of this research are consistent with other published studies, further research is needed to assess other risk factors associated with ART.

\section{PERIOD OF PURPLE CRYING PROGRAM FOR THE PREVENTION OF ABUSIVE HEAD TRAUMA/SHAKEN BABY SYNDROME}

Edmond Power*, Farhana Sharif. School of Medicine, University College Dublin

\subsection{6/archdischild-2021-europaediatrics.476}

To review instances of abusive head trauma in Ireland and the United Kingdom To discuss the benefits of the Period of Purple Crying program To begin the pathways to implement the Period of Purple Crying program in Ireland.

Research published since the beginning of the COVID-19 pandemic has shown a marked increase in the instances of abusive head trauma in the United Kingdom. While there are many contributing factors to abusive head trauma, in these cases one of the main precipitants was the enforced isolation during the pandemic. An educational program is needed now more than ever to highlight the drastic consequences of abusive head trauma in infants and educate parents on what level of crying is to be expected from infants.

Areas that have implemented the Period of Purple Crying program have seen a reduction in the numbers of abusive head traumas. Other benefits of the program included an increased awareness among parents of what to do when a baby is crying. Cost-of-illness analysis also shows that the implementation of a program can have massive cost-saving benefits.

Ireland does not currently have a preventative program for shaken baby syndrome. The National Healthy Childhood Programme includes child health screen and developmental surveillance and would be an ideal means by which to introduce the Period of Purple Crying program. This service provides for parent contact with healthcare professionals at least twenty-five times beginning in pregnancy and ending at the child's third birthday. More research is also needed regarding exact numbers of abusive head trauma in Ireland.

\section{TOXIC STRESS AND NEURODEVELOPMENTAL TRAJECTORIES - OPORTUNITY FOR INTERVENTION}

Vanja Slijepčević Saftićc. Child and Youth Protection Center of Zagreb

10.1136/archdischild-2021-europaediatrics.477

Objective The effects of negative environmental factors in childhood result in neuro-biological changes. Exposed to powerful, negative environment, lead to hyperactivation of the neurological, imune and endocrine system. This state is known as a toxic stress. Symptoms may frequently imitate ADHD and other neurodevelopmental disorders. Hyper-vigilance and dissociation, could be mistaken for inattention. Impulsivity might be brought on by a stress response.

Method The aim of this study was to estimate preliminary results of a multi-disciplinary (Pediatric neurologist, Social worker, Psychologist, Psychiatrist, Speech therapist and special need expert) clinical assessment of patient came to Child and Youth protection Center, Zagreb, Croatia, in case of adverse childhood experiences, acording ACEs (Adverse Childhood Experience score) $\geq 4$. From 2015 - 2021 in Child and Youth Protection Center Zagreb we prospectively observed 1954 children with $\geq 4$. We were looking for presence of minor neurological dysfunctions (gross motor function, mild dysfunction in posture, reflexes, coordination, fine manipulative ability, fine motor disfunction, dyskinesia and excessive associated movements). For those with ACES $\geq 4$, and neurodevelopmental delay, EEG was indicated. We also evaluated simptoms of impulsivity, hyperactivity, and an inability to focus.

Results Neglect, physical and emotional abuse and high conflict divorce are the most important adverse experiences resulting in deviations in neurodevelopment. Impulsive behaviour, and other form of ADHD 'like' variants are significantly more frequent (over 50\%) in children with history of toxic stress. Over $30 \%$ of patients with ACES $\geq 4$ showed non-specific changes in EEG. In case of foetal abuse (Neonatal Abstinence Syndrome) 19 percent showed cognitive impairment, over 50 percent symptoms from ADHD spectrum, 33 percent had speech problems, and over 60 percent had learning problems. Child faced with toxic stress are obese in more than 70 percent.

Early detection of adverse childhood experiences help us to start with early interventions in order to prevent short and long term consequences of Toxic stress. 